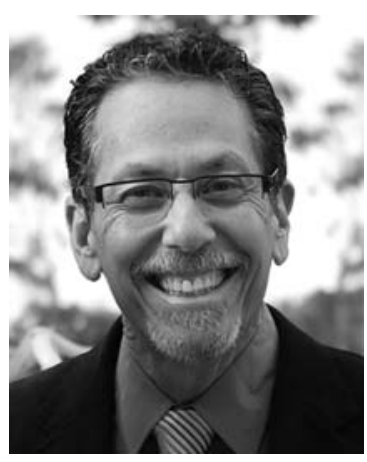

\title{
A Conversation with Ron Evans
}

\author{
INTERVIEWER: RICHARD SEVER \\ Executive Editor, CSH Perspectives and CSH Protocols
}

Ron Evans is Director of the Gene Expression Laboratory at the Salk Institute for Biological Studies and a Howard Hughes Medical Institute Investigator.

Richard Sever: The vitamin D receptor is a member of a family of nuclear receptors that you discovered 30 years ago. Can you tell us in general terms about nuclear receptors and what distinguishes them from cell surface receptors?

Dr. Evans: In typical hormonal mechanisms, a ligand binds to the cell surface, activates a kinase on the intracellular side, and then uses a series of cascades to transmit an effect within the cell. In contrast, nuclear receptors reside in the nucleus, where they directly exert their effect by interacting with and controlling entire networks of genes. The receptor finds specific sites in chromatin and either unlocks or represses those sites. They control complex physiologic responses by literally marshaling hundreds of genes to be activated and hundreds to be repressed. The receptors respond to ligands, so you can have a highly regulated network that is tuned to a specific physiologic challenge like a high-fat diet, or starvation, or changes in cholesterol. Many aspects of our physiology are controlled by changing the gene activity in the nucleus this way. It's a fundamentally different type of signal transduction, but it's very effective and serves a specific role that is different from pathways that use cell surface receptors.

Richard Sever: What distinguishes the ligands for this type of receptor compared with other receptors?

Dr. Evans: Nuclear receptor ligands are typically small molecules that more often than not are metabolites of the pathway that the receptor controls. For example, bile acids are involved in digestion, but they're also a functional ligand for nuclear receptors. The receptors that respond to bile acids control bile acid metabolism. Most of the ligands for nuclear receptors are lipids, so they can get through the lipid bilayer. Fatty acids and cholesterol derivatives are typical ligands. They get into the cells and control pathways that regulate the metabolism of those molecules themselves.

Richard Sever: Steroids are also ligands for some nuclear receptors.
Dr. Evans: Steroids are forms of cholesterol modified in particular ways that allow them to be a little bit more soluble. Cholesterol is very hydrophobic, and the steroids are chemically modified to be able to interact with receptors. Steroids control their own production, and their release from organs such as the adrenals or gonads is typically controlled by either metabolic stress or neuronal signaling. There's a network of these hormones circulating through our blood. Because of their chemical nature, they're permeant to every tissue, including the brain. No tissue is free from being fully exposed to steroids, and as a result they can control neural activity and visceral activity simultaneously, and thus coordinate physiological responses.

Richard Sever: Does this ability to hit every cell provide a higher order of coordinated response or combinatorial action?

Dr. Evans: Yes, but in an unexpected way. The nuclear receptor takes the ligand directly to the gene itself - or the network of genes, really - that is being regulated, but the genes it affects are going to be different in every cell type. If there are 200 cell types in the body, there will be 200 gene expression programs that are simultaneously activated. That's what controls and coordinates this physiologic response. It uses transcription as the core underpinning of the physiologic mechanism.

Richard Sever: Each nuclear receptor has two regions: the DNA-binding region and the ligand-binding region. These are not separated. In other signaling molecules, you see domain shuffling during evolution where different modules have paired with other modules. Why doesn't that happen with nuclear receptors?

Dr. Evans: There are only two transcription factor families that did not evolve in bacteria or yeast. Nuclear receptors are one, and ETS (E26 transformation-specific) transcription factors are the other. The DNA-binding domain and the ligand-binding domain of the nuclear receptor are unique structural motifs. They're common to all animals - we don't know of any exceptions - but 
not present in plants or any other type of organisms; thus, it's a metazoan-specific motif. We think that the origin of these physiologic mechanisms resides in the novel motif of the receptor itself. It provided a new way of signaling. The ligand-binding domain allows it to sense chemicals, but the unique DNA-binding domain actually allows it to control transcription.

Richard Sever: One of the chemicals that is sensed is vitamin D. Vitamin D receptors start showing up in injured liver and pancreatic tissue, correct?

Dr. Evans: Vitamin D was isolated in 1917 and it was the fourth vital factor identified after vitamins A, B, and C. We've known about it for 100 years, but how it actually works is only just being deciphered. It principally works through the vitamin $\mathrm{D}$ receptor that binds to a very specific nucleotide sequence. We thought we knew what it does, which was control calcium, a very important process in bone mineralization and bone remodeling. What we also discovered is that the normal liver has no real levels of the vitamin D receptor, but when a liver is injured the receptor levels increase more than 100-fold. That high level of expression comes from a particular cell that's involved in wound healing called the stellate cell. When liver tissue is damaged, the wound healing response gets activated. Normally, that cell expands during the healing process, takes care of the wound, and when the damage signal - a molecule called TGF- $\beta$ goes away the whole system regresses to a quiescent state.

Under chronic injury, such as a viral or parasitic infection or a cancer, that acute response doesn't turn off; it's like a wound that won't heal. The tissue is continually inflamed, there's a continual fibrotic response, and the liver ultimately progresses to cancer. The same process happens in the pancreas. This response is sometimescalled a stromal response. The surrounding cells - not the cancer itself but the cells trying to fight off the canceractually encourage the cancer. It's like a living "skin" around the tumor. The activated cells, the wound-healing cells, produce an impressive number of collagen molecules. Eighteen different collagens are deposited in the environment of the tumor. They also produce $\mathrm{T}$-cell blockade molecules like CXCL12 (C-X-C motif chemokine 12 ) that prevent the immune system from invading the tumor, and powerful cytokines like interleukin 6 (IL6) that often are linked with cancers during their active stage of expansion.

If you provide the vitamin D ligand, you turn off the chronic response from those cells. Vitamin D acts like a molecular brake. It very effectively and relatively rapidly turns off literally hundreds of genes, including all the collagen genes, the T-cell blockade genes, and the IL6 genes. Each one of those by themselves would be a benefit, but turning them all off provides a multipathway effect by giving just one simple chemical. That's the advantage of targeting a nuclear receptor: You affect hundreds of genes, as opposed to just using an antibody to IL6 or CXCL12 that would only take out that one component. We're taking out those components and hun- dreds more that complement them, and we turn off the entire process for a window of time. The window of time allows drugs and immune cells to reach the tumor, and that's the real advantage. The "skin" opens up, drugs can rush in, and the autoimmune T-cell response can penetrate and immunologically try to remove the tumor. In a combinatorial setting, the effectiveness of already approved drugs is dramatically higher with vitamin D. It's a synergizer, a simple molecule that enhances existing therapies. Vitamin D doesn't actually kill the tumor. It allows the tumor to be killed.

Because the vitamin D ligand is not itself present in the environment of the tumor, we supply it either as a potent injectable form or as a specialized pill that is much more potent than what you'd take as a vitamin supplement. We're having great success using this simple molecule as a new therapeutic approach to treating pancreatic cancer. The science is actually interestingly complex, but the therapeutic approach is deceptively simple. Clinical trials have already started. Patients are being treated at a number of different hospitals and we're beginning to evaluate the therapy, which is looking very promising.

Richard Sever: But you can't just take large doses of vitamin D and hope for the best?

Dr. Evans: You could, but taking a supplement is just the beginning of getting the active molecule. You need a lot of good sun exposure. If you're very disciplined you can do that, but you have to be careful because the supplement can also give rise to high calcium. Of course, if you have pancreatic cancer, you might not care about that. However, the synthetic derivative that we recommended is actually relatively inexpensive. It's also much safer and more effective. It's an already approved drug, but not for cancer therapy. It typically takes a long time to get FDA approval for new cancer treatments, but because we are using an already approved drug, we're hoping it'll be quicker.

Richard Sever: Is this one of those vitamins that people should actually be taking every morning before breakfast?

Dr. Evans: The average person is not vitamin D-deficient. On the other hand, most people who are sick are vitamin D-deficient. Chronic illness leads to a progressive lowering of vitamin D levels in the body, and that reduction increases your risk for something happening somewhere. We believe that the lower level of vitamin $\mathrm{D}$ that's seen in many diseases is a reflection of this wound healing response that consumes the body's vitamin D supplies. When they are consumed, it's hard for that response to turn off. The body can be resupplied using our drug-Zemplar, or paricalcitol-by injection or taking a pill, and that goes directly to the sites of deficiency and reverses this process. It's actually a little bit more complex than that, but basically all of the vitamin $\mathrm{D}$ that we're returning to the body goes to the right spot almost immediately. It goes to where the levels are lowest and fills up that bucket first. As a result, you get a very quick response, literally within days. 
Richard Sever: There's many cancers, many wounds that don't heal. You talked about fibrosis, various other conditions that are chronic. Are you looking at other conditions on which to follow up?

Dr. Evans: Every new kind of therapy needs a start, but most cancer drugs find uses in other cancers. The next ones that we'd look at would be kidney, liver, colon, lung, breast. They all have a fibrotic response. There's no literature to suggest it's the same kind of response that we see in liver and pancreas, but we believe that in fact it is. If we're correct, then vitamin D should be very effective in the same way to improve the therapies used to treat these other diseases. 


\section{$\$_{\text {CSH\& }}^{\infty}$ Cold Spring Harbor Symposia SYMPOSIA On Quantitative Biology}

\section{A Conversation with Ron Evans}

Cold Spring Harb Symp Quant Biol 2015 80: 318-320

Access the most recent version at doi:10.1101/sqb.2015.80.030080

\section{License}

Email Alerting Receive free email alerts when new articles cite this article - sign up in Service the box at the top right corner of the article or click here. 